Culture et histoire dans l'espace roman

$6 \mid 2011$

Figures du pouvoir dans la littérature hispanoaméricaine

\title{
Le pouvoir et son envers dans A.B.U.R.T.O d'Heriberto Yépez
}

«Carlitos » vs « El Subcomandante »

\section{Anaïs Fabriol}

\section{(Q) OpenEdition Journals}

Édition électronique

URL : https://journals.openedition.org/cher/9649

DOI : $10.4000 /$ cher.9649

ISSN : 2803-5992

\section{Éditeur}

Presses universitaires de Strasbourg

\section{Édition imprimée}

Date de publication : 30 juin 2011

Pagination : 127-136

ISBN : 978-2-35410-033-9

ISSN : $1968-035 X$

\section{Référence électronique}

Anaïs Fabriol, «Le pouvoir et son envers dans A.B.U.R.T.O d'Heriberto Yépez », reCHERches [En ligne], 6 | 2011, mis en ligne le 17 décembre 2021, consulté le 24 janvier 2022. URL : http:// journals.openedition.org/cher/9649; DOI : https://doi.org/10.4000/cher.9649 


\title{
Le pouvoir et son envers dans A.B.U.R.T.O d'Heriberto Yépez «Carlitos » vs «El Subcomandante»
}

\author{
Anaïs FabRIOL \\ Université de Caen Basse-Normandie
}

$\mathrm{D}$ ans son roman A.B.U.R.T.O., publié en 2005, Heriberto Yépez s'attache à décrire le processus menant l'ouvrier Mario Aburto à assassiner, le 23 mars 1994, le candidat du $\mathrm{PRI}^{1}$ à la présidence du Mexique, Luis Donaldo Colosio. Le récit, agencé de manière post-moderne autour de la figure du tueur, a donc toutes les raisons d'introduire une réflexion sur le rapport au pouvoir, apanage du PRI en cette fin de $\mathrm{XX}^{\mathrm{e}}$ siècle.

Or, si des figures du pouvoir transparaissent ça et là par petites touches dans les premiers chapitres, ce n'est qu'à partir du quatrième (sur un total de cinq), Todas tus tragicomedias, tres o cuatro fantasmas (p. 101-158) que le texte va poser deux portraits suivis d'individus censés s'opposer dans le Mexique de l'époque: le président de la République, Carlos Salinas de Gortari (soupçonné d'être le commanditaire du meurtre de Colosio), et le Sous-Commandant Marcos, alors porte-parole de la guérilla zapatiste. Tous deux sont exposés presque parallèlement, par touches successives et à travers le regard quasi goguenard d'un narrateur externe dont le lecteur ne connaîtra jamais vraiment l'identité - mais qui semble être une projection de Yépez lui-même. Ce parallélisme, auquel s'ajoute l'absence totale de la figure de Colosio, la victime, semblent tendre vers une mise en perspective de la notion même de pouvoir, et partant, de tout ce qui peut le composer dans la société mexicaine contemporaine: faillite des idéaux

1 Partido Revolucionario Institucional, au pouvoir de 1929 à l'an 2000. 
révolutionnaires, mainmise du PRI sur la sphère politique, disparition progressive de l'idéologie nationaliste qui prévalait jusqu'alors; le fait que le ton employé soit ouvertement celui de la satire marque la déconstruction des grands mythes politiques du $\mathrm{XX}^{\mathrm{e}}$ siècle.

Carlos Salinas, que l'on trouve surtout dans le texte sous le nom de Carlitos, est introduit dès le début du chapitre dans une histoire hallucinée que Mario Aburto semble raconter à la police, où se mêlent morts et vivants et où il reproche à la curandera María Sabina d'avoir donné la recette du peyotl aux gringos et autres ennemis de la Patrie. A la fin de l'entretien, il déclare: «Sí, Salinas. El fue el que mató a María Sabina². Era su sirvienta. [...] A ella se le dijo: Tú eres una vieja puerca. Eres basura y luego la mató Salinas ${ }^{3}$ » (Yépez 2005: 107). A la page suivante, le narrateur extérieur introduit l'enfance du personnage, puis son adolescence et son arrivée au pouvoir (p. 112 sq), enfin, son mandat comme président. Dans la dernière partie du chapitre, il est montré de manière surréaliste, commandant une armée de «chupacabras» et désireux de trouver une méthode pour tuer son successeur pour se représenter à une présidentielle - ce qui, selon la loi électorale mexicaine, est strictement impossible. Ce qui entraîne une scène totalement délirante, aux accents de mauvais trip, où l'un de ses "chupacabras» lui ramène Mario Aburto, à qui il commandite le meurtre de son successeur.

Le Sous-commandant Marcos, quant à lui, apparaît uniquement dans le dernier tiers, juste avant que Carlitos ne se décide à tuer Colosio. Une fois de plus, c'est Mario Aburto qui l'introduit dans le texte, via une diatribe réservée à sa nouvelle conquête, la très cynique Gabriela: "no dudo que haya conocido al subcomandante Marcos. No lo dudo ni tantito. Yo he ido a reuniones de ésas en todo el país» (134). Un bref portrait est alors dressé, mais ce n'est qu'à la suite du passage où le narrateur externe accuse Salinas du meurtre de Colosio et le représente en train de le fomenter, qu'un portrait plus complet du Sous-commandant sera proposé.

Bien que celui de Carlos Salinas soit plus étoffé, les deux portraits présentent de nombreux points communs. A commencer par le fait qu'ils soient intéressés dès le début par le pouvoir politique, et que ce soit, parmi toutes les figures qui hantent le roman, celles qui ne semblent mues que

2 María Sabina communiqua les différentes formes de l'utilisation du peyotl à Carlos Castañeda et à Fernando Bénitez dans les années 1960.

3 Une rumeur circulait au Mexique dans les années 1990, selon laquelle Carlos et Raúl Salinas, adolescents, auraient abattu l'une des domestiques de leurs parents, sans avoir été inquiétés pour ce crime. 
par un désir d'être à la tête d'autres hommes, et ce, pour camoufler un défaut physique. Il semblerait que sur ce point, Yépez les mette sur un pied d'égalité, en insistant sur des détails anatomiques particulièrement ridicules et sur la soif de pouvoir, comme s'ils étaient intimement liés. Ainsi, au sujet de Carlitos, le narrateur externe dira d'emblée :

Como todos los niños mexicanos, Carlitos deseaba convertirse, algún día, en presidente. Solamente que el sueño de Carlitos no era tan delirante como el del resto de millones de escuincles en el país. Carlitos era parte de las familias en el poder, una de las más importantes. Así que para algún día convertirse en presidente no tenía que competir con otros millones de ilusos, sino con unas cuantas decenas de familias pertenecientes al Partido Revolucionario y a las compañías en el poder. [...] En la escuela primaria, Carlitos siempre fue el más chaparro de los niños (108-109).

Plus loin, il définira même les défauts physiques de Salinas comme un choix conscient pour accéder au pouvoir et le conserver:

Carlos tenía el cuerpo de Hitler combinado con el de Cantinflas. Ése era el personaje que había elegido. Lo había elegido porque estaba convencido, como Foucault, que entre más irrisorio el dirigente, más inmune se vuelve. Más era la confirmación de su poder, porque si un dirigente es un bufón o rey Ubu, el poder que ejerce es más incuestionable, pues aún entre las manos de un pequeño monstruo -un payaso pusilánime-,un payaso pobre, el poder es ejercido hasta sus últimas (138).

En ce qui concerne Marcos, on a un processus similaire, légèrement plus compacté dans la mesure où moins de texte lui est consacré, mais qui en tout cas suit les mêmes lignes directrices:

Cuando menos lo pensaba, Marcos se había hecho un guerrillero. Toda su vida había estado obsesionado con los relatos heroicos, con matar a los que nos jodían. Llegar primero que todos. Encabezar la historia. Desde la preparatoria, a Marcos le gustaba charlar con sus compañeros y demostrarles que era el más radical. Seguramente no se le paraba bien o la tenía chiquita y por eso tenía que andar metido en cosas de la izquierda y pendejadas de ésas (140).

Autant Marcos que Carlos sont donc présentés comme des êtres recherchant le pouvoir comme manière de pallier certaines humiliations, des défauts physiques cachés ou patents. Dans le cas de Carlos, ils sont évidents, assumés, et lui ont apparemment permis de parvenir au pouvoir. Dans le cas de Marcos, on se trouve face à un défaut sexuel - et, partant, narcissique? -, caché, non apparent, qui est la cause initiale de ses délires révolutionnaires. On remarquera, de plus, que tout ce qui concerne le rapport au physique chez 
Marcos est bien évidemment caché, comme si l'image de l'homme masqué devait perdurer par-delà le récit. Et si l'on en croit la perfide Gabriela, «si [los Zapatistas] no se quitan siquiera sus mascaritas es porque han de estar súper feos» (135). Autant Carlos que Marcos sont définis comme des personnages ayant une certaine inadaptation sociale et qui se sont servis de cette inadaptation pour construire leur pouvoir, que ce soit à la tête du parti le plus puissant du pays ou d'une guérilla paysanne dans l'État le plus pauvre de la République.

Il est intéressant de voir que Yépez ne se pique aucunement de considérations idéologiques, mais place bien les deux personnages dans le même panier: l'un veut se retrouver à la tête de l'histoire, coûte que coûte, et l'autre devenir le président du pays. Le pouvoir les définit; et il semble également les constituer. Sans doute est-ce possible dans un pays, d'où d'après le narrateur externe, gouverner est aller au fond de l'infamie:

Gobernar en México significa burlar. Poner en la cima de la pirámide, en la presidencia misma, al más evidente payaso, al más vulgar de la tribu para así dejar claro que el poder, incluso en las manos de Tribilín o el Marqués de Sade interpretado por Luis de Alba, va a ser un poder terrible y ejecutado (139).

Si ce passage peut s'appliquer uniquement à Carlitos et à la manière dont il semble envisager la politique, il parait également que cela vise tout Mexicain cherchant à détenir un quelconque pouvoir, qu'il soit officiel ou contestataire, de gauche ou de droite. Si celui qui gouverne via le PRI est un "payaso", un mélange de personnage de Disney et de sadisme, le révolutionnaire à la Marcos, n'est guère épargné dans son délire: "en la prepa, [Marcos] se infectó de ideas izquierdosas [...] en que te arriesgas a convertirte en un mero farsante. $Y$ es que el lenguaje de la izquierda marea. Cuando menos te das cuenta ya estás en la derecha» (108). Du «farsante» au "payaso", n'est-on pas toujours dans une idéologie du faux, dans une construction grossière visant à tromper le public, le faire rire sur des leurres et des stratégies narratives/discursives souvent peu raffinées, afin qu'il concède le pouvoir sans se poser de questions idéologiques au dernier à l'avoir harangué? En outre, la vision du politique est plus que désabusée (quelles que soient les idéologies exprimées, elles finissent toujours par se rejoindre): l'emploi de "se infectó", "marea" renvoient à une sorte de maladie, une maladie qui abolirait le discernement et fausserait tous les rapports, notamment entre celui qui détient le pouvoir et ceux qui le soutiennent - ou s'y opposent. 
De plus, les «ideas izquierdosas» se situent comme un contrepoint au pouvoir du PRI, dont on serait bien en peine de déterminer la couleur politique dans les années 1990, tant il semble mu par l'unique volonté de conserver le pouvoir; le flou généré par le PRI se répercute sur son opposant médiatique, opposant non-légitime dans le sens où il se construit comme guérilla et sans respecter le jeu "démocratique». Carlos comme Marcos se situent donc dans un univers où le pouvoir est flou, déterminé par des actes délirants ou crapuleux, et où ils semblent régner sur leurs propres fantasmes avant de commander qui que ce soit. C'est à travers la représentation de la vacuité du pouvoir que se dessine un autre parallèle entre les deux hommes: celui de l'illégitimité de leur position. Carlos est certes, de par son appartenance à l'oligarchie liée au PRI, un gouvernant en partie légitime. Il n'empêche que l'infamie de son arrivée au pouvoir est martelée :

[Salinas] había llegado al poder gracias a un fraude electoral cometido contra un ex miembro del Partido revolucionario, Cuauhtémoc Cárdenas, el hijo del Tata Lázaro, el presidente mexicano más popular del siglo XX [...] y que para llegar al poder había formado un partido de izquierdas, pero apenas Carlitos se consolidó en la silla presidencial le prometió todo al país, jel Primer Mundo!, y se convirtió por un periodo en el presidente más popular de todos (114).

Ce qui l'oblige à se justifier devant Mario dans les termes suivants «Llegué a la presidencia gracias a un fraude, ¿y qué? Tú llegaste a este mundo gracias a otro, tu madre, así que cállate» (145). Il est étrange de voir d'ailleurs que Carlos, qui ne semble pas s'embarrasser du respect de la loi, se résout finalement à assassiner Colosio parce que celui-ci refuse de faire modifier la constitution pour lui permettre d'être réélu. Mais par cette saillie s'exprime sans doute une des idées directrices du fragment: le pouvoir est pathétique et transforme en personnage satirique celui qui tombe sous son emprise.

Le même processus a bien évidemment lieu avec Marcos, sous forme d'un dialogue entre les Indiens de la guérilla zapatiste:

- Dice que hay que transcender las divisiones entre nosotros, que hay que llegar a la sociedad mexicana con este mensaje.

- ¿Quiere ser vocero, o qué?

- Ándale, algo así.

- Bien, dile que bienvenido, que él sea el que salga en la televisión. Pero déjale bien claro que con esta jeta no va a representarnos. Dile que se ponga capucha (142). 
Marcos est donc contraint de se travestir pour arriver à devenir le guérillero qu'il a toujours rêvé d'être. De plus, son personnage finit par être un double travestissement, puisqu'il devient son propre héraut, serviteur du vrai Marcos, celui qui semble avoir le pouvoir sur les autres guérilleros, et partant, sur lui-même: "Marcos era el subcomandante, el verdadero comandante estaba dentro de su mente. Marcos estaba a las órdenes del Otro Marcos» (141).

Ici se niche une différence de taille entre Salinas et Marcos: le premier n'est qu'une créature de pouvoir, construite à travers celui-ci et doté de tous les attributs de l'autocrate latino-américain; son seul but est clairement de posséder le pouvoir et ses émanations, qu'elles soient financières ou diplomatiques, quel que soit le prix à payer. Le second semble construire à travers le pouvoir une autre forme de création, littéraire celle-ci. Yépez le souligne à plusieurs reprises: «Marcos se ha vuelto uno de sus heterónimos. Marcos siempre ha querido ser un pinche escritor y ya» (142); le pouvoir, pour le personnage, semble avoir été une médiation vers sa vraie vocation, la littérature. Une littérature clairement orientée vers l'intertextualité - "cuando escribía sus cartas o declarados, en ellos mezclaba historias tojolabales, monsivaísmo, fraseología que recordaban los manifiestos dadaístas y estridentistas y poemas suyos mezclados con letras de Joaquín Sabina o trova cubana» (135) -, ce qui semble montrer à quel point le personnage de Marcos est composite et influencé par d'autres figures de la littérature de centre-gauche mexicaine et latino-américaine.

En revanche, la figure de Carlitos est tout entière dédiée au pouvoir; c'est d'ailleurs cette orientation qui, au sein de l'économie narrative du texte, tient lieu de fil rouge entre les différents fragments qui lui sont consacrés. Contrairement à Marcos, dont l'ascension au pouvoir ne contient que le vague épisode fondateur de son "infection" aux idées gauchistes, pour reprendre, en les traduisant, les propos de Yépez, Carlitos possède, quand à lui, tout un paragraphe dédié à son enfance et au moment où il a décidé d'être celui qui prendrait le pouvoir, au détriment de son frère Raúl. A la manière d'un tableau naïf, il y est représenté en compagnie de Miguel de La Madrid, son prédécesseur ${ }^{4}$, de Raúl et d'une série de personnages politiques des années 1970-1980.

La scène est ouvertement satirique et métaphorique : Salinas et La Madrid, présents sous les noms de Carlitos et Miky, enfants (aucune précision n'est

4 Président du Mexique de 1982 à 1988. 
donnée, sinon qu'ils sont à l'école primaire), s'amusent à humilier un de leurs camarades, Manuelito. Manuelito doit, entre autres, deviner qui, de La Madrid ou de Salinas, est son véritable père; il ne trouve jamais la bonne réponse et se fait donc constamment rabrouer. Parallèlement, dans la même école existe un autre gang d'enfants, les Súper Popis ${ }^{5}$, dont fait partie Raúl, mais qui n'entre pas en rivalité avec les précédents car, comme nous le dit Yépez: «Junto con Miky, Carlitos se dedicaba a mandar a Manuelito. [...] El poder de los Súper Popis no les preocupaba » (109). Dès le départ, la relation possible avec les Súper Popis s'inscrit dans la possibilité d'une lutte pour le pouvoir, une lutte où l'enjeu est certes faible (la possession de Manuelito), mais une lutte qui semble amuser Carlitos et Miky puisqu'ils ne peuvent s'en passer. D'une certaine manière, Manuelito préfigure déjà le PRI, puis le Mexique: ces choses sur lesquelles La Madrid et Salinas sont appelés, sans encore le savoir probablement, à régner.

Évidemment, comme tout épisode d'enfance, celui-ci doit amener sa blessure narcissique et la leçon qu'en tire le personnage - voire le narrateur -, et il n'y a là aucune exception à la règle:

Y enviar [a Manuelito] para que lo reprendieran los Súper Popis fue una broma pesada que hermanó a los Súper Popis con Carlitos y Miky. [...] El resto de la escuela sabía que Carlitos y Miky deseaban ascender al poder y que, una de dos, estaban retando a los Súper Popis o deseaban fortalecer sus vínculos con ellos. [...]

Los Súper Popis no entendían el juego de Carlitos. Fue Raulín, su hermano, que se acercó a Manuelito y le preguntó qué se traían aquellos dos, y Manuelito le explicó que tenía que adivinar quien era su padre. [...]

- Entonces, tarado, diles que tu padre soy yo- le ordenó Azueta. Grítales eso. [...]

- Mi padre no es ninguno de los dos. Mi padre es Azueta.

Toda la escuela oyó eso. A partir de ese día Manuelito pasó a ser parte de los Súper Popis. El juego les había salido mal. Este día, Carlos y Miky supieron que no tenían escapatoria. O pasaban al poder o el poder iba a pasar sobre ellos (110-111).

La leçon des Súper Popis porte ses fruits: tous deux seront présidents de la République, se disputant non plus Manuelito, mais tout le pays:

Cuando Miky, a su vez, entregó la banda presidencial tricolor, lo hizo en el cuerpo de Carlitos.

[...] Carlitos y Miky, desde la altura de aquella posición superior, volteaban a mirar al país entero y una pregunta atravesó la mente de ambos.

«¿Ahora quién es tu padre?» (113).

5 Popis: snob, appartenant à la haute société. 
L'épisode des Súper Popis semble vouloir montrer que la soif de pouvoir réside finalement dans peu de chose: le désir de posséder des esclaves heureux de l'être et disposés à se faire humilier à tout moment. Manuelito pourrait s'en aller et refuser l'humiliation que lui imposent ses deux bourreaux, or il ne le fera que lorsqu'il tombera sur plus puissant qu'eux et qu'il assistera à leur propre humiliation. La réflexion de Yépez est finalement très hégélienne: l'esclave satisfait (ici Manuelito, puis le peuple mexicain, soutenant dans un premier temps Salinas qui lui a promis la prospérité) est autant responsable que son maitre de la situation injuste dans laquelle il se trouve. Manuelito est content de son sort car on se dispute son esclavage. Le peuple est heureux car on lui promet des cuisines et des séries télévisées. Dans les deux cas, tous deux sont acteurs de leur prison. Ce qui n'empêchera pas le premier de trahir, et les seconds de souhaiter la mort de Salinas à la fin de son mandat: le narrateur affirmera que "ese año todos queríamos tronar la cabeza de la Gran Rata» (143)6. Comme dit le texte, ou l'on accède au pouvoir ou il nous écrase.

L’omniprésence des termes liés au pouvoir - «poder», «mandar» - et leur lien direct avec une filiation hasardeuse marquent le côté fabuleux de l'épisode; on se trouve dans une sorte de genèse du personnage de Carlos, une sorte de genèse où le personnage a déjà tous ses attributs crapuleux: il propose de l'argent à un professeur pour ne pas aller en cours, exploite sans vergogne Manuelito et a une relation teintée de rivalité avec son meilleur - et seul - ami, Miky. Le fait que ces personnages soient représentés des années après, adultes, en pleine passation de pouvoir, montre bien combien ils ont été façonnés par celui-ci; c'est du reste, comme nous avons pu le dire ou le voir plus tôt, l'unique raison d'être de Carlos, dont on nous dira d'ailleurs que le père a fait plusieurs fils dans le seul but d'en voir un accéder à la magistrature suprême. L'épisode de Manuelito montre bien combien le personnage n'est orienté que par cette quête: ses jeux d'enfant ne sont que le reflet de ce qu'il va devenir: un homme de pouvoir, tout simplement.

C'est d'ailleurs cet épisode fondateur qui justifie le reste de la construction du texte et de la réflexion sur le pouvoir. Puisque Carlos a été programmé pour obtenir le pouvoir, après l'épisode des Súper Popis il ne peut se définir autrement, d'où ses plans de réélection et le meurtre final de Colosio.

6 Car désormais il est devenu impopulaire et le peuple le compare à «una rata», soit, d'après le jeu de mots intraduisible, à la fois à un rat et à un voleur. 
C'est dans cette dimension que les personnages de Marcos et de Carlos diffèrent le plus. Pour Marcos - ce qui peut paraître étrange pour une figure qui a été présentée comme «marxista más bien ortodoxo »-, le pouvoir n'est qu'une médiation pour parvenir à autre chose, l'écriture. Salinas, quant à lui, est entièrement défini par la notion de pouvoir. Qu'il le perde et il n'est plus rien, d'ailleurs il n'est pas représenté après son départ du pouvoir, ce qui tombe sous le sens.

Mais dès lors, pourquoi les avoir présentés en parallèle? Ce qui semble transparaître, au fond, c'est que les épisodes qui parsèment la narration sont avant tout construits comme émanant de rumeurs publiques, le narrateur écrivain déclarant lui-même:

Pero cada vez que una persona pregunta a otra quién fue, todos opinan que la orden vino de Salinas. Yo soy un escritor. Soy la persona que menos fe tiene en la verdad. No me culpen de las versiones que ahí anoto. Estas versiones no las inventé yo. Son las que cuenta un país y yo, acaso, les saco punta o las caricaturizo. No me interesa relatar la verdad. Solo me interesa dejar claro que como todos ustedes, pertenezco al narcorrealismo (137). ${ }^{7}$

On est donc dans une stratégie narrative d'assemblage, de recompilation, qui ne serait pas novatrice si le pacte de lecture n'arrivait pas a posteriori, un peu après la moitié du roman. Le lecteur, jusque là, a cru avoir affaire à une biographie d'assassin postmoderne, à la manière du Libra de Don De Lillo (1988) - ce qui déjà présuppose une bonne série de ruptures avec le canon narratif classique -, or, il se retrouve maintenant au milieu d'un montage de rumeurs et de suppositions où même la véracité diégétique est malmenée. Les figures de Carlos et de Marcos sont accompagnées de celles $\mathrm{du}$ "chupacabras $»^{8}$, grande invention de la presse aux ordres du pouvoir de la fin du $\mathrm{XX}^{\mathrm{e}}$ siècle pour cacher les récits de corruption, de malversation ou tout simplement l'essor de la guérilla zapatiste. Or, cette allusion nous renvoie à ce que Christian Salmon décrit dans Storytelling (2007), à savoir la mainmise sur la narration des faits politiques aux États-Unis des spin doctors:

[Les spin doctors] fabriquent des histoires afin de conquérir le pouvoir puis de l'exercer et prennent en charge leur diffusion virale, créent l'horizon d'attente dans lequel elles vont prendre consistance, manipulent les conditions sociotechniques de leur diffusion et de leur interprétation (Salmon 2007 : 123).

7 Cette apparition de l'écrivain-narrateur vient en quelque sorte en écho à l'avertissement au lecteur qui précède l'incipit: «Además los hechos reales nunca lo fueron» (8).

8 Vampire qui, dans le Nord du Mexique, sucerait le sang du bétail. Dans les années 90, il n'était pas rare de le voir faire la une de journaux nationaux à grand tirage. 
Or, cette prise de pouvoir des spin doctors, des histoires sur l'Histoire, est selon Christian Salmon, l'un des tournants marquants de la conception du pouvoir aux États-Unis dans les années 1990. De là à supposer que de telles techniques aient également été employées au Mexique à la même période pour couvrir les faits marquants (crise économique, corruption, puis bien évidemment meurtre de Colosio, moins anodin à l'époque qu'il ne le paraitrait maintenant), il n'y a qu'un pas que Yépez semble présupposer d'emblée. Le récit est défini plusieurs fois comme « un chisme», "un chiste»; Salinas parle de Marcos comme de son «empleado» (144), Marcos vit dans ses récits, où il possède plus de pouvoir que dans la vie réelle: tout porte à croire que Yépez, comme il le revendique d'ailleurs, a agencé sa diégèse comme le conglomérat de toutes les rumeurs. Le chapitre étudié dans cet article pourrait d'ailleurs être vu comme une variation sur le thème du récit de pouvoir, mais un récit morcelé, à l'allure de miroirs déformants, où chacun, de Marcos, Carlos, voire Mario Aburto, croit être le chef d'orchestre mais ne possède finalement en lieu et place de conducteur qu'une partition tronquée.

En fin de compte, les figures présentes ici, et construites autour du pouvoir, ne sont pas à proprement parler des personnages au sens classique du terme. Le Sous-commandant Marcos et le Carlos Salinas de Gortari qui apparaissent sont plutôt les figures d'un jeu de massacre, une sorte de collage de tout ce qui s'est publié ou s'est dit sur eux, à tort ou à raison, à un moment M circa 1994. Leur rapport au pouvoir sert à augmenter leurs facettes les plus satiriques, à en faire ces caricatures que l'on voit dans les journaux ou dont parlent leurs inféodés, un peu partout, à l'usine, dans le «colectivo», dans les restaurants. En quelque sorte, et puisque Yépez fait référence ouvertement à Jarry: Ubu roi est bien de retour en la personne de Salinas, et son pouvoir va si loin qu'il teinte toute la partie qui lui est consacrée dans A.B.U.R.T.O, influençant aussi la caricature de son principal opposant. Même si celui-ci a plus que le pouvoir politique: il a celui de l'écriture.

\section{Bibliographie}

Bénitez, Fernando, 1970, Los Indios de México, México, Era.

De Lillo, Don, 1988, Libra, Londres, Penguin Books.

Salmon, Christian, 2007, Storytelling, Paris, La Découverte.

Yépez, Heriberto, 2005, A.B.U.R.T.O, México, Random House Mondadori. 